\title{
Number Used at Visit
}

National Cancer Institute

\section{Source}

National Cancer Institute. Number Used at Visit. NCI Thesaurus. Code C157390.

An indication of the number of drugs used during the visit. 